\title{
Clinical characteristics and treatment of critically ill patients with COVID-19 in Hebei
}

\author{
Yuhong Chen ${ }^{1}$, Kun Zhang ${ }^{1}$, Guijun Zhu ${ }^{1}$, Lixia Liu ${ }^{1}$, Xixin Yan ${ }^{2}$, Zhigang Cai ${ }^{2}$ Zhongheng Zhang ${ }^{3}$, \\ Haijun $\mathrm{Zhi}^{4}$, Zhenjie $\mathrm{Hu}^{1} \wedge$ \\ ${ }^{1}$ Department of Intensive Care Unit, Hebei Medical University Fourth Affiliated Hospital and Hebei Provincial Tumor Hospital, Shijiazhuang, \\ China; ${ }^{2}$ Department of Respiration, Hebei Medical University Second Affiliated Hospital, Shijiazhuang, China; ${ }^{3}$ Department of Emergency \\ Medicine, Sir Run Run Shaw Hospital, Zhejiang University School of Medicine, Hangzhou, China; ${ }^{4}$ Emergency Department, Cangzhou Central \\ Hospital, Cangzhou, China \\ Contributions: (I) Conception and design: Y Chen, Z Hu; (II) Administrative support: Health commission of Hebei province; (III) Provision of study \\ materials or patients: Y Chen; (IV) Collection and assembly of data: Y Chen, K Zhang; (V) Data analysis and interpretation: K Zhang, Y Chen, H \\ Zhi, Z Zhang; (VI) Manuscript writing: All authors; (VII) Final approval of manuscript: All authors. \\ Correspondence to: Prof. Zhenjie Hu, MD; Yuhong Chen, MD. Department of Intensive Care Unit, Hebei Medical University Fourth Affiliated \\ Hospital and Hebei Provincial Tumor Hospital, 12 Jiankang Road, Shijiazhuang, China. Email: syicu@vip.sina.com; yuhong_apple@126.com.
}

Background: In December, 2019, a novel coronavirus disease 2019 (COVID-19) emerged in Wuhan, China. We aimed to clarify the epidemiology, laboratory examinations, imaging findings, and treatment of critically ill patients with COVID-19 in Hebei province, China.

Methods: In this retrospective study, the demographic, laboratory and imaging, and treatment data of patients with severe COVID-19 treated in 13 designated hospitals in Hebei were collected and analyzed.

Results: A total of 319 severe COVID-19 patients were treated at the 13 designated hospitals between 22 January, 2020 and 25 March, 2020. Eventually, 51 critically ill (31 severe cases and 20 critically severe cases) patients were included in the analysis. The patients had an average age of $58.9 \pm 13.7$ years, and $27(52.9 \%)$ were men. Twenty-one (41.2\%) were familial cluster, and 33 (64.7\%) had chronic illnesses. The patients in critically severe group had longer duration from symptom to confirmation, more severe infections, more severe lung injury, and a lower percentage of lymphocytes. All 51 patients received antiviral drugs, 47 (92.2\%) received antibacterial agents, $49(96.1 \%)$ received traditional Chinese drugs, and $46(90.2 \%)$ received methylprednisolone. The critically severe patients received more fluid and more diuretic treatment; 14 (70.0\%) required invasive mechanical ventilation, and 13 (65.0\%) developed extrapulmonary complications.

Conclusions: COVID-19 patients who had underlying diseases and longer confirmation times were more likely to progress to critically severe COVID-19. These patients also presented with a higher risk of respiratory depression, circulatory collapse, extrapulmonary complications, and infection.

Keywords: 2019 novel coronavirus; novel coronavirus disease 2019 (COVID-19); SARS-CoV-2; 2019-nCoV; critically ill

Submitted May 09, 2020. Accepted for publication Jul 09, 2020.

doi: 10.21037/apm-20-1273

View this article at: http://dx.doi.org/10.21037/apm-20-1273

\footnotetext{
^ ORCID: 0000-0003-1404-5691.
} 


\section{Introduction}

In December 2019, an outbreak of pneumonia of unknown etiology emerged in Wuhan, the capital city of Hubei province in China. Eventually, the pathogen was confirmed to be a distinct clade of Betacoronavirus and officially named as severe acute respiratory syndrome coronavirus 2 (SARS-CoV-2); the disease was termed novel coronavirus disease 2019 (COVID-19) (1,2). As of 25 March, 2020, the disease had infected 413,467 people and killed 18,433 (4.46\%) people in 186 countries.

According to Chinese Clinical Guidelines for COVID Pneumonia Diagnosis and Treatment (7th edition) COVID-19 cases are classified as mild, moderate, severe, or critically severe (3). Patients in Hubei experienced relatively severe symptoms and had a high mortality rate compared to patients in other Chinese provinces (4), which may be attributed to the demand for health care outstripping available resources (5). Early identification and efficient treatment are of paramount importance to reducing mortality among patients with severe COVID-19. Herein, we describe the epidemiological clinical features and treatment of patients with severe COVID-19 in Hebei province. Our experience may be of considerable value to the early identification and efficient treatment of patients with a high risk of progression to critically severe COVID-19. We present the following article in accordance with the STROBE reporting checklist (available at http:// dx.doi.org/10.21037/apm-20-1273).

\section{Methods}

\section{Study design and population}

A multicenter, retrospective, observational trial of critically ill patients with COVID-19 who received treatment at 13 designated hospitals in Hebei, China, between 22 January, 2020 and 25 March, 2020, was conducted. The patients were diagnosed by SARS-CoV-2 nucleic acid real-time polymerase chain reaction (RT-PCR) and the local health authority were categorized as severe or critically severe cases based on the Chinese Clinical Guidelines for COVID Pneumonia Diagnosis and Treatment ( $7^{\text {th }}$ edition) (3). Cases meeting any of the following criteria were categorized as a severe: (I) respiratory distress: respiratory rate $>30$ breaths per minute; (II) percutaneous oxygen saturation $\left(\mathrm{SpO}_{2}\right)$ $<93 \%$ when resting; and (III) partial pressure of oxygen $\left(\mathrm{PaO}_{2}\right) /$ fraction of inspired oxygen $\left(\mathrm{FiO}_{2}\right) \leq 300 \mathrm{mmHg}$. Cases meeting any of the following criteria were categorized as critically severe: (I) respiratory failure requiring mechanical ventilation; (II) shock; (III) other organ failure, and needed monitoring and treatment in intensive care unit (ICU). Patients aged $\leq 18$ years, patients with a hospital stay of $\leq 48$ hours, and pregnant patients were not included. All procedures performed in this study involving human participants were in accordance with the Declaration of Helsinki (as revised in 2013). Informed consent was taken from all the patients. This study was approved by the Ethics Commission of the Fourth Clinical Medical College of Hebei Medical University (2020KS002).

\section{Data collection}

Patient data, including demographic information, exposure history, underlying diseases, symptoms from onset to hospital admission, vital signs upon confirmation of the disease type, chest computed tomography (CT) images, laboratory values on categorization of the disease type, the Sequential Organ Failure Assessment (SOFA) score, the Acute Physiology and Chronic Health Evaluation II (APACHE II) score, treatment, and extrapulmonary comorbidities, were collected. Any missing data were obtained and uncertain records were clarified through direct communication with the managing physician.

\section{Statistical analysis}

Continuous variables with a non-normal distribution were described as median (interquartile range, IQR) and analyzed with the nonparametric Mann-Whitney $\mathrm{U}$ test (6). Continuous variables with a normal distribution were expressed as the mean (standard deviation), and groups were compared using $t$-tests. Categorical variables were expressed as count (percentage) and compared with Pearson's chisquare test or Fisher's exact probability test. All statistical analyses were performed using IBM SPSS Statistics version 26.0. A two-sided $\mathrm{P}$ value of $<0.05$ was considered to be statistically significant.

\section{Results}

\section{Epidemiological characteristics}

By 25 March 2020, 319 cases of COVID-19 had been recorded in Hebei, including $56(17.55 \%)$ critically ill patients. Of them, 51 patients ( 31 severe cases and 20 critically severe cases) were eventually included in this study. 


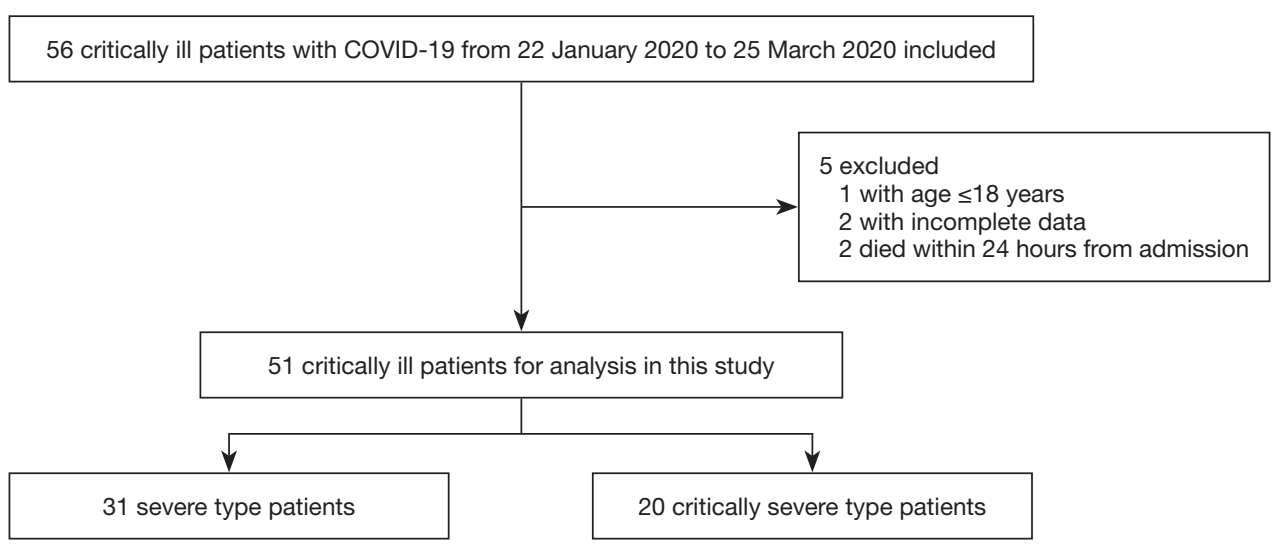

Figure 1 Study flow diagram.

The other 5 patients were excluded for the following reasons: aged $\leq 18$ years $(n=1)$, lack of clinical data $(n=2)$, and death within 24 hours (n=2) (Figure 1). The patients had an average age of $58.9 \pm 13.7$ years old, and $27(52.9 \%)$ were men. None of the patients had a history of exposure to Wuhan's Huanan seafood market. The majority (78.4\%) of patients had a known contact history, and more than half $(51.0 \%)$ were familial clusters (Table 1). There were 33 (64.7\%) patients who had chronic illnesses including hypertension (21 cases, $41.2 \%$ ), cardiovascular disease (12 cases, $23.5 \%$ ), diabetes (11 cases, $21.6 \%$ ), and pulmonary disease (8 cases, $15.7 \%)$. Few patients had other diseases or a smoking history. Sixteen (31.4\%) patients had a surgical history (Table 1).

\section{Symptoms from onset to hospital admission}

The most common symptoms experienced by patients were fever (94.1\%), cough (66.7\%), expectoration (37.3\%), dyspnea $(35.3 \%)$, fatigue $(27.5 \%)$, myalgia $(13.7 \%)$, and diarrhea (11.8\%); however, headaches $(7.8 \%)$ were rarely reported (Table 2). The duration from symptom onset to admission, symptom onset to laboratory confirmation, and symptom onset to classification was 3.0 (range, 1.0-6.0), 6.0 (range, 3.0-10.0), and 10.2 \pm 4.7 days, respectively. The differences in these durations between the two groups were not statistically significant $(\mathrm{P}>0.05)$ (Table 2). Compared to the severe group, a higher proportion of patients in the critically severe group had a confirmation time of longer than 10 days $(\mathrm{P}=0.010)$.

\section{Vital signs, and laboratory and imaging findings}

The critically severe group had a higher maximum daily temperature and respiratory rate than the severe group, but lower blood pressure. Therefore, vasoconstrictive agents were more frequently used in critically severe patients $(12$, $60.00 \%)$ (Tables 3,4).

The critically severe group had lower $\mathrm{PaO}_{2} / \mathrm{FiO}_{2}$ $(<150 \mathrm{mmHg})$ and received higher $\mathrm{FiO}_{2}$ levels to maintain $\mathrm{SpO}_{2} \geq 93 \%$ than the severe group (Table 3). Both groups had a mild elevation of lactate concentration but without significant difference. The white blood cell (WBC) count, percentage of neutrophils, and the levels of C-reactive protein and procalcitonin of the critically severe group were significantly elevated compared with those of the severe group; conversely, the percentage of lymphocytes was significantly lower $(\mathrm{P}<0.05)$ (Table 3$)$. The critically severe group also had a higher erythrocyte sedimentation rate (ESR) than the severe group, but the difference between the groups was not statistically significant $(\mathrm{P}>0.05)$ (Table 3$)$. Additionally, the critically severe patients had mild coagulation disorders with slightly prolonged prothrombin time (PT), and slightly elevated levels of blood urea nitrogen $(\mathrm{BUN})$ and creatine kinase $(\mathrm{P}<0.05)$; as a result, their APACHE II and SOFA scores were higher than those of the severe patients (Table 3).

The counts of cluster of differentiation CD4+, CD8+, and $\mathrm{CD} 3+$ cells were measured in 13 patients (5 critically severe cases and 8 severe cases), and the ratio of $\mathrm{CD} 4+$ to CD8+cells (CD4+/CD8+) was calculated.

Patients with critically severe disease tended to have lower levels of CD4+ cells and CD4+/CD8+, but the difference between the groups was not statistically significant $(\mathrm{P}>0.05)$ (Table 3).

In the severe group, all but $2(96.1 \%)$ patients had bilateral infiltrates on chest CT, and most patients showed 
Table 1 Demographics and baseline characteristics of critically ill patients with COVID-19

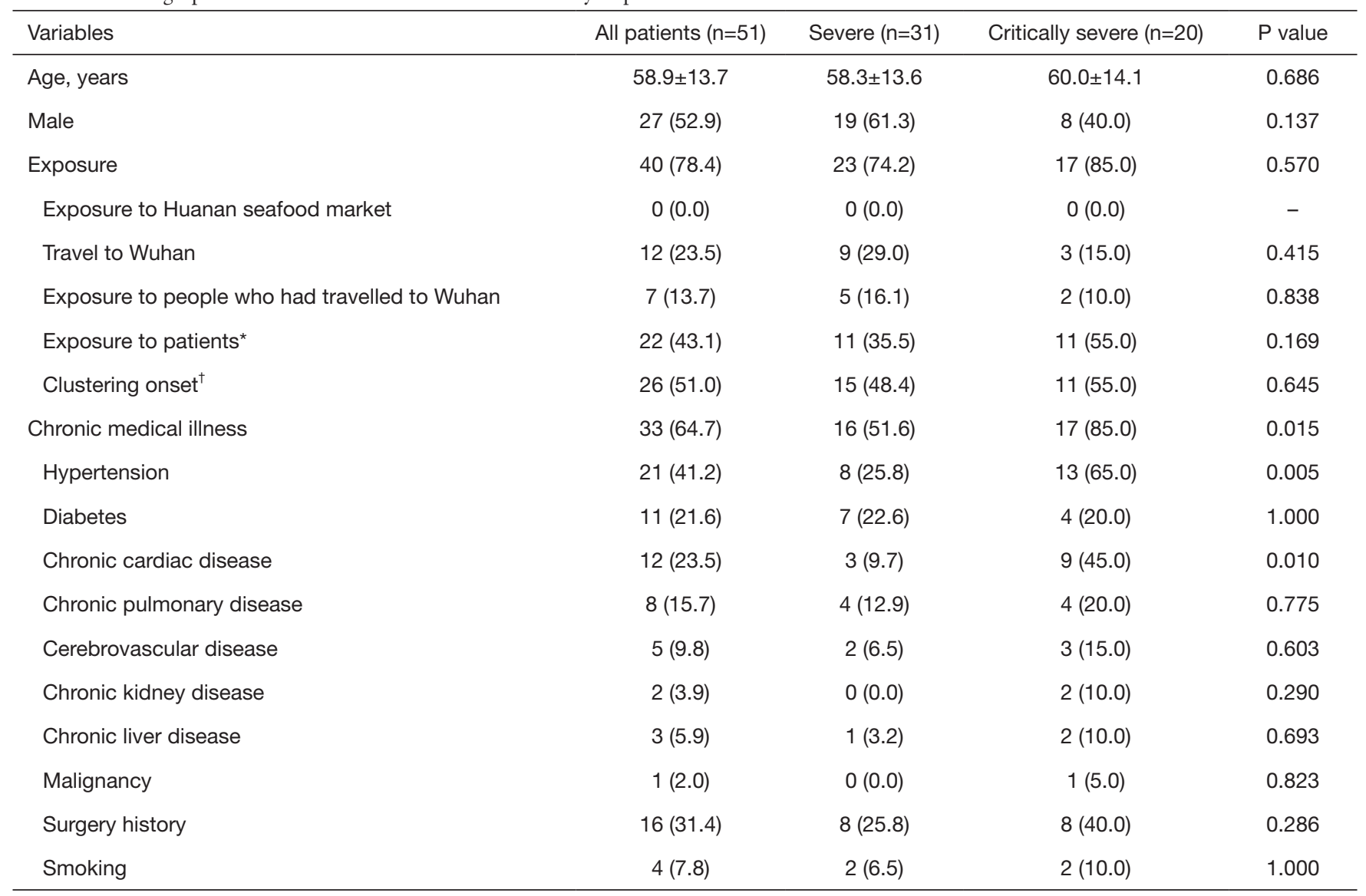

The results were described as mean (standard deviations) or counts (percentages). ${ }^{\text {, }}$ patients who have confirmed COVID-19 infection or are highly suspected of being infected; ${ }^{\dagger}, 2$ or more cases of fever and/or respiratory symptoms within 2 weeks in enclosed spaces such as the home, office, school/classroom, etc. COVID-19, novel coronavirus disease 2019.

localized infiltrates. In the critically severe group, chest CT demonstrated diffuse, extensive interstitial, and alveolar infiltrates (Figure 2), making these patients more likely to experience shortness of breath (Table 2).

\section{Treatment, extrapulmonary comorbidities, and outcomes}

All of the patients were treated with antiviral therapy: $50(98.0 \%)$ with interferon, 49 (96.1\%) with lopinavir/ ritonavir, $37(72.5 \%)$ with arbidol, and $11(21.6 \%)$ with oseltamivir). Many of the patients (47, 92.2\%) were also treated with antibacterial agents, including azithromycin, levofloxacin, moxifloxacin, cefoperazone sodium, sulbactam sodium/tazobactam sodium, and piperacillin sodium and tazobactam sodium (Table 4). Combined antibiotic therapy was more commonly received by the critically severe patients than the severe patients. Methylprednisolone was used to treat $46(90.2 \%)$ patients, and the treatment duration tended to be longer in the critically severe group $(\mathrm{P}=0.095)$. Approximately one-half of the patients were treated with the immunizing agents Xuebijing and albumin, $49(96.1 \%)$ were treated with traditional Chinese drugs, and approximately one-quarter received blood transfusion (Table 4).

All participants developed acute respiratory distress syndrome (ARDS), and the patients with critically severe COVID-19 had more severe dyspnea and lower oxygenation than the patients with severe disease (Table 3). In the severe group, $23(74.2 \%)$ patients were treated with a nasal cannula, and $6(19.4 \%)$ received a high-flow nasal cannula. In the critically severe group, $14(70.00 \%)$ patients were treated with invasive mechanical ventilation, $11(55.0 \%)$ with prone-position ventilation, 4 (20.0\%) with noninvasive mechanical ventilation, $3(15.0 \%)$ with continuous renal 
Table 2 Symptoms of critically ill patients with COVID-19 from onset to hospital admission

\begin{tabular}{|c|c|c|c|c|}
\hline Variables & All patients $(n=51)$ & Severe $(n=31)$ & Critically severe $(n=20)$ & $P$ value \\
\hline Fever & $48(94.1 \%)$ & $30(96.8 \%)$ & $18(90.0 \%)$ & 0.693 \\
\hline Cough & $34(66.7 \%)$ & $18(58.1 \%)$ & $16(80.0 \%)$ & 0.105 \\
\hline Expectoration & $19(37.3 \%)$ & $11(35.5 \%)$ & 8 (40.0\%) & 0.745 \\
\hline Myalgia & 7 (13.7\%) & $5(16.1 \%)$ & $2(10.0 \%)$ & 0.838 \\
\hline Fatigue & $14(27.5 \%)$ & $8(25.8 \%)$ & $6(30.0 \%)$ & 0.743 \\
\hline Diarrhea & $6(11.8 \%)$ & $3(9.7 \%)$ & $3(15.0 \%)$ & 0.896 \\
\hline Headache & $4(7.8 \%)$ & $3(9.7 \%)$ & $1(5.0 \%)$ & 0.942 \\
\hline Confirmation time $\geq 10 \mathrm{~d}$ & $13(25.5 \%)$ & $4(12.9 \%)$ & 9 (45.0\%) & 0.010 \\
\hline Duration from symptom onset to classification, days & $10.2 \pm 4.7$ & $9.8 \pm 5.0$ & $10.9 \pm 4.1$ & 0.301 \\
\hline No. of positive COVID-19 nucleic acid tests, times & $1.0(1.0,1.0)$ & $1.0(1.0,1.0)$ & $1.0(1.0,1.0)$ & 0.656 \\
\hline
\end{tabular}

The results were described as median (interquartile ranges), mean (standard deviations), or counts (percentages), as appropriate. COVID-19, novel coronavirus disease 2019.

Table 3 Vital signs and laboratory and imaging findings of critically ill patients with COVID-19 within 24 hours of the final clinical classification (severe or critically severe type)

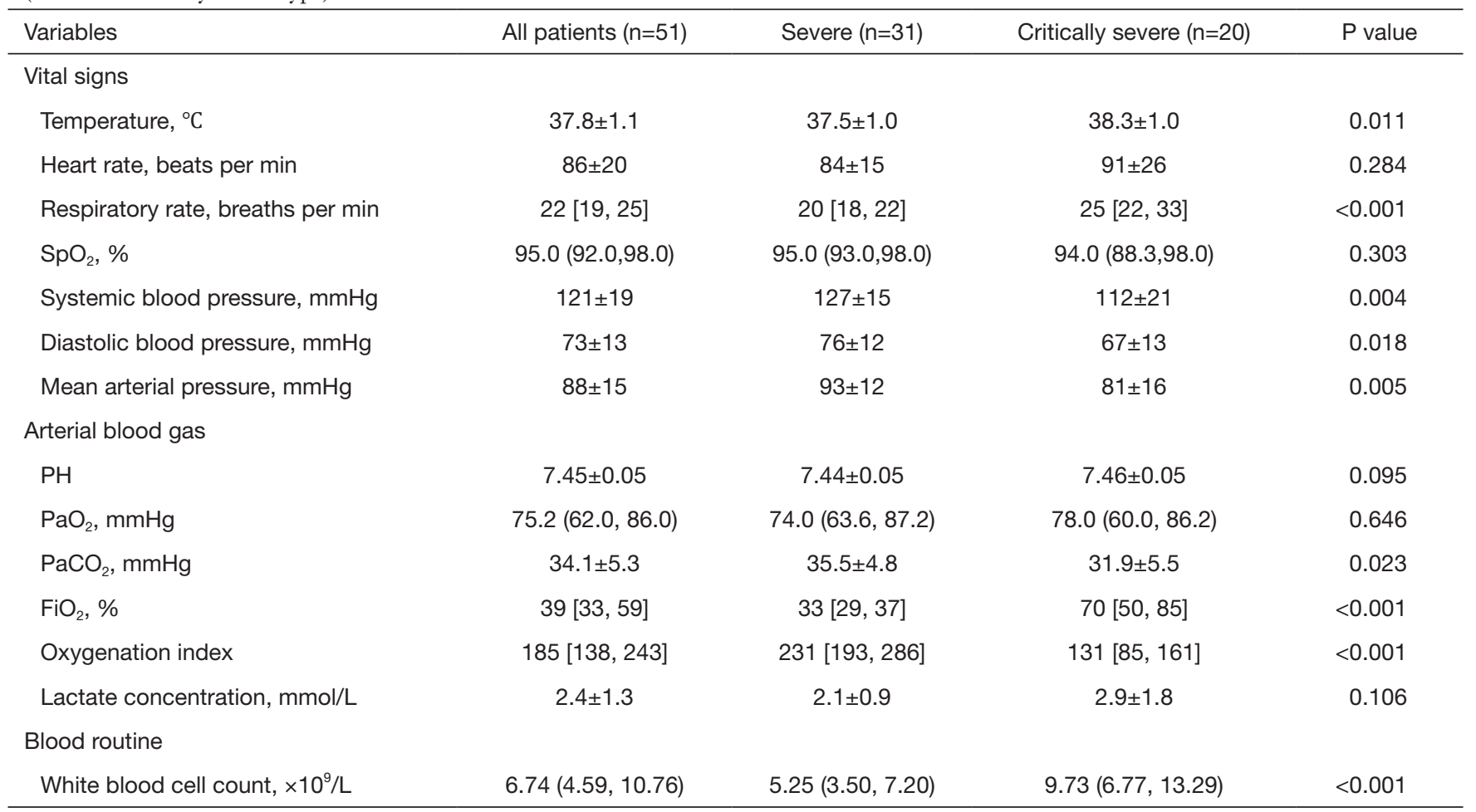

\footnotetext{
Table 3 (continued)
} 
Table 3 (continued)

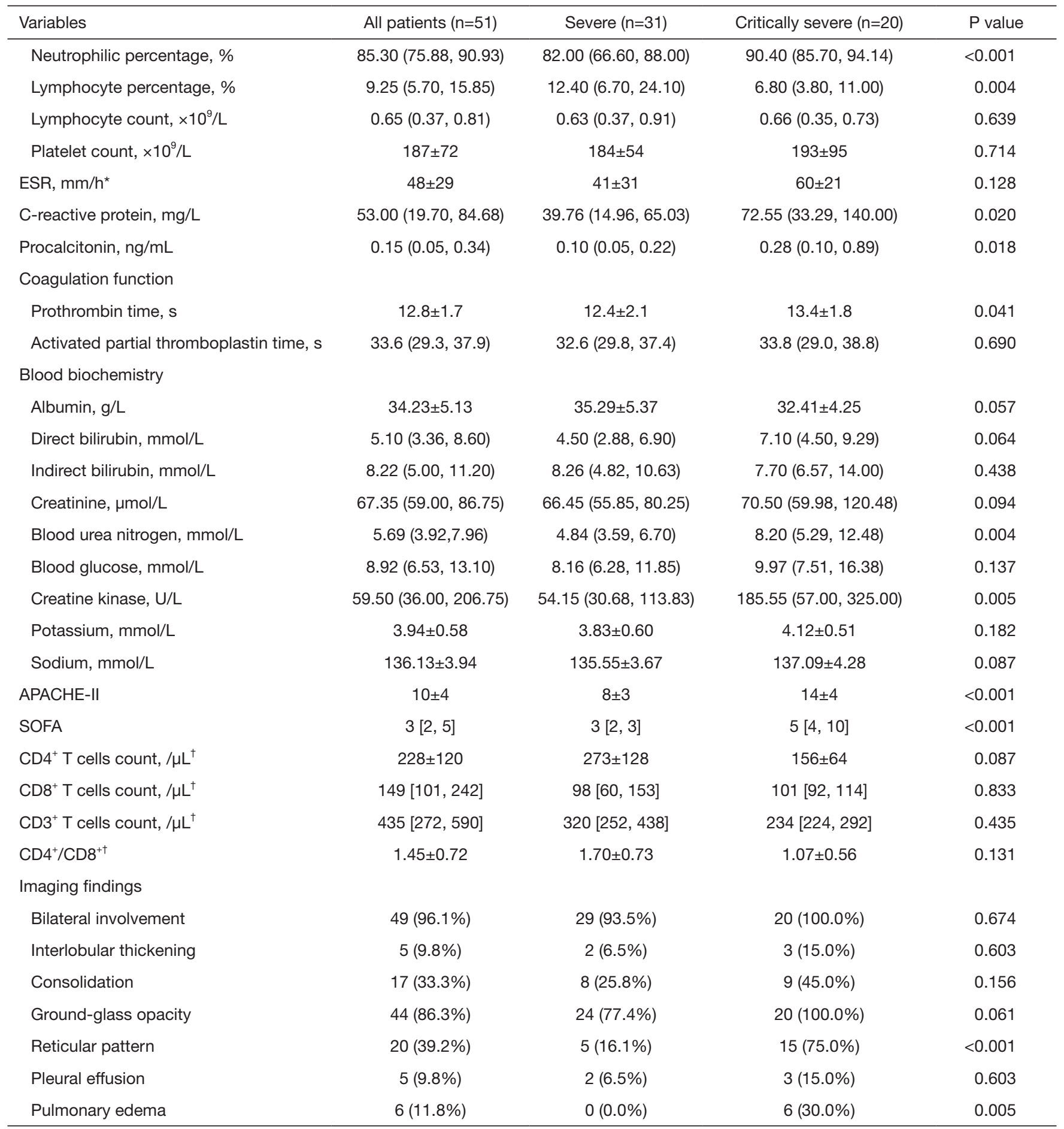

The results were described as median (interquartile ranges), mean (standard deviations), or counts (percentages), as appropriate. *, data available for 24 patients; ${ }^{\dagger}$, data available for 13 COVID-19 patients (8 severe cases and 5 critically severe cases). COVID-19, novel coronavirus disease 2019; $\mathrm{SpO}_{2}$, percutaneous oxygen saturation; $\mathrm{PaO}_{2}$, arterial oxygen pressure; $\mathrm{PaCO}_{2}$, partial pressure of carbon dioxide; $\mathrm{FiO}_{2}$, oxygen concentration; ESR, erythrocyte sedimentation rate; $\mathrm{CD}$, cluster of differentiation; APACHE-II, Acute Physiology and Chronic Health Evaluation II; SOFA, Sequential Organ Failure Assessment. 
Table 4 Treatments, extrapulmonary comorbidities, and outcomes of critically ill patients with COVID-19

\begin{tabular}{|c|c|c|c|c|}
\hline Variables & All patients $(n=51)$ & Severe $(n=31)$ & Critically severe $(n=20)$ & $P$ value \\
\hline Nasal cannula & $23(45.1 \%)$ & $23(74.2 \%)$ & $0(0.0 \%)$ & - \\
\hline High-flow nasal cannula & $8(15.7 \%)$ & $6(19.4 \%)$ & $2(10.0 \%)$ & - \\
\hline Non-invasive mechanical ventilation & $6(11.8 \%)$ & $2(6.5 \%)$ & $4(20.0 \%)$ & - \\
\hline Prone position ventilation & $11(21.6 \%)$ & $0(0.0 \%)$ & $11(55.0 \%)$ & $<0.001$ \\
\hline ECMO & $2(3.9 \%)$ & $0(0.0 \%)$ & $2(10.0 \%)$ & 0.290 \\
\hline CRRT & $3(5.9 \%)$ & $0(0.0 \%)$ & $3(15.0 \%)$ & 0.107 \\
\hline Antiviral treatment & $51(100.0 \%)$ & $31(100.0 \%)$ & $20(100.0 \%)$ & - \\
\hline Arbidol & $37(72.5 \%)$ & $23(74.2 \%)$ & $14(70.0 \%)$ & 0.743 \\
\hline Interferon & $50(98.0 \%)$ & $31(100.0 \% 19)$ & $19(95.0 \%)$ & 0.823 \\
\hline Antibiotic treatment & $47(92.2 \%)$ & $28(90.3 \%)$ & $19(95.0 \%)$ & 0.942 \\
\hline Combined use of antibiotics & & & & $<0.001$ \\
\hline Single antibiotic treatment & $18(35.3 \%)$ & $17(54.8 \%)$ & $1(5.0 \%)$ & - \\
\hline Combination of two antibiotics & $13(25.5 \%)$ & 7 (22.6\%) & $6(30.0 \%)$ & - \\
\hline Combination of three or more antibiotics & $16(31.4 \%)$ & $4(12.9 \%)$ & $12(60.0 \%)$ & - \\
\hline Antifungal treatment & $4(7.8 \%)$ & $0(0.0 \%)$ & $4(20.0 \%)$ & 0.039 \\
\hline \multicolumn{5}{|l|}{ Glucocorticoids } \\
\hline Intravenous immunoglobulin therapy & $28(54.9 \%)$ & $13(41.9 \%)$ & $15(75.0 \%)$ & 0.021 \\
\hline Human albumin & $26(51.0 \%)$ & $11(35.5 \%)$ & $15(75.0 \%)$ & 0.006 \\
\hline Blood transfusion & $15(29.4 \%)$ & $2(6.5 \%)$ & $13(68.4 \%)$ & $<0.001$ \\
\hline Thymalfasin & $27(52.9 \%)$ & $14(45.2 \%)$ & $13(65.0 \%)$ & 0.166 \\
\hline Diuretic & $29(56.9 \%)$ & $12(38.7 \%)$ & $17(85.0 \%)$ & 0.001 \\
\hline Xuebijing injection & $30(58.8 \%)$ & $18(58.1 \%)$ & $12(60.0 \%)$ & 0.891 \\
\hline Traditional Chinese medicine & $49(96.1 \%)$ & $30(96.8 \%)$ & $19(95.0 \%)$ & 1.000 \\
\hline rhBNP & $6(11.8 \%)$ & $0(0.0 \%)$ & $6(30.0 \%)$ & 0.005 \\
\hline Levosimendan & $5(9.8 \%)$ & $0(0.0 \%)$ & $25(10.0 \%)$ & 0.014 \\
\hline Vasoactive drugs & $12(23.5 \%)$ & $0(0.0 \%)$ & $12(60.0 \%)$ & $<0.001$ \\
\hline Dopamine & $7(13.7 \%)$ & $0(0.0 \%)$ & 7 (35.0\%) & 0.002 \\
\hline
\end{tabular}

Table 4 (continued) 
Table 4 (continued)

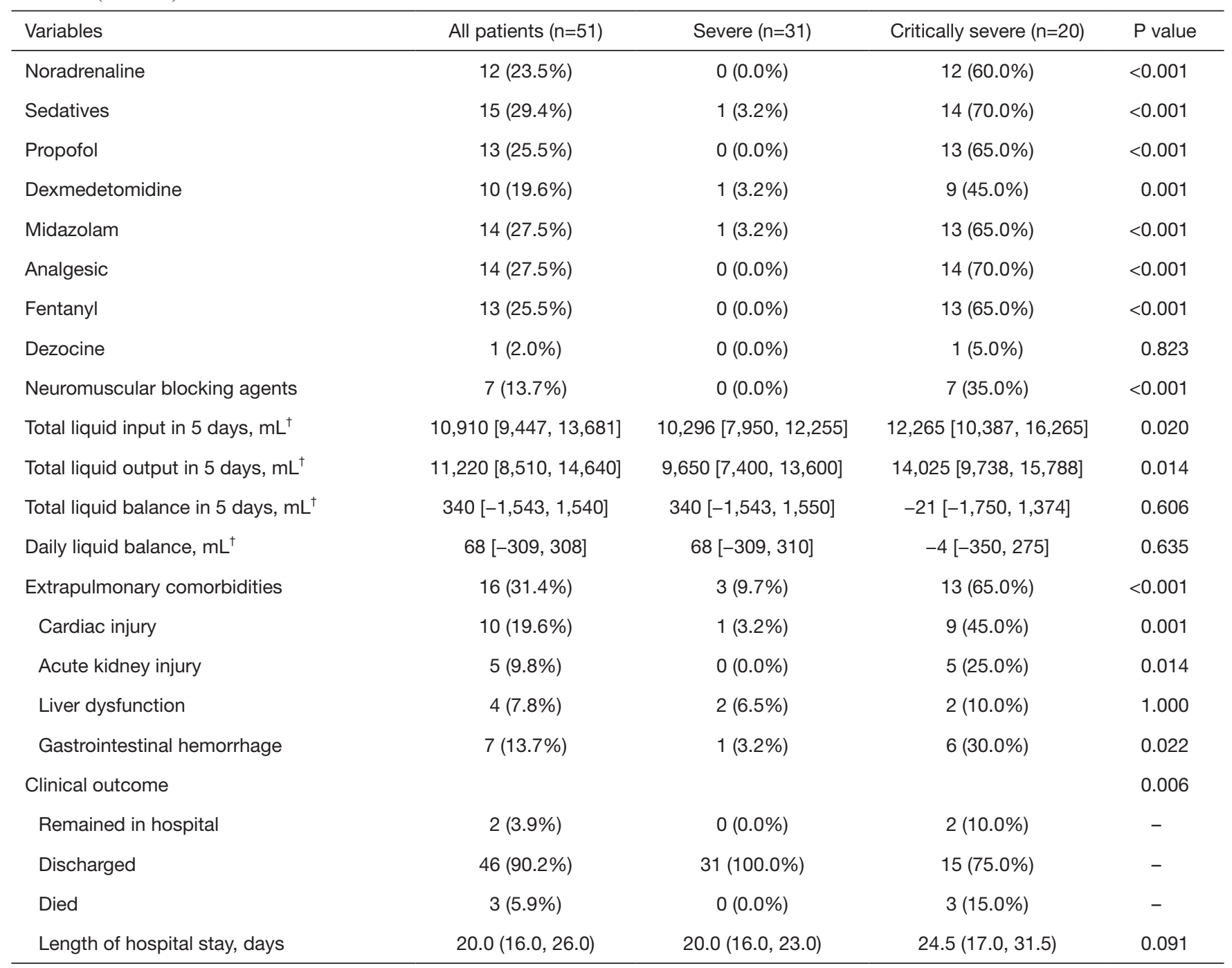

The results were described as median (interquartile ranges), mean (standard deviations), or counts (percentages), as appropriate. *, data available for 38 patients; ${ }^{\dagger}$, data available for 47 patients. CRRT, continuous renal replacement therapy; ECMO, extracorporeal membrane oxygenation; rhBNP, lyophilized recombinant human brain natriuretic peptide.

replacement therapy (CRRT), $2(10.0 \%)$ with a high-flow nasal cannula, and $2(10.0 \%)$ with extracorporeal membrane oxygenation (ECMO) therapy (Table 4). The critically severe group, more cases received sedation, analgesics, vasoconstrictors, fluid and diuretics than in the severe group (Table 4).

Thirteen $(65.0 \%)$ patients with critically severe disease had extrapulmonary organ injury: $9(45.0 \%)$ had cardiac injury, 6 (30.0\%) had gastrointestinal bleeding, 5 (25.0\%) had acute kidney injury, and $2(10.0 \%)$ had liver dysfunction (Table 4). The critically severe patients had worse clinical outcomes. Only 3 (15.0\%) patients died in the critically severe group. The length of hospital stay was longer in the critically severe group, but the difference between the groups was not statistically significant $(\mathrm{P}=0.091)$.

\section{Discussion}

Since December, 2019, COVID-19 has spread around the world, carrying with it considerable morbidity and mortality. The management of severe patients is the key point of treatment. We described the epidemiological, 

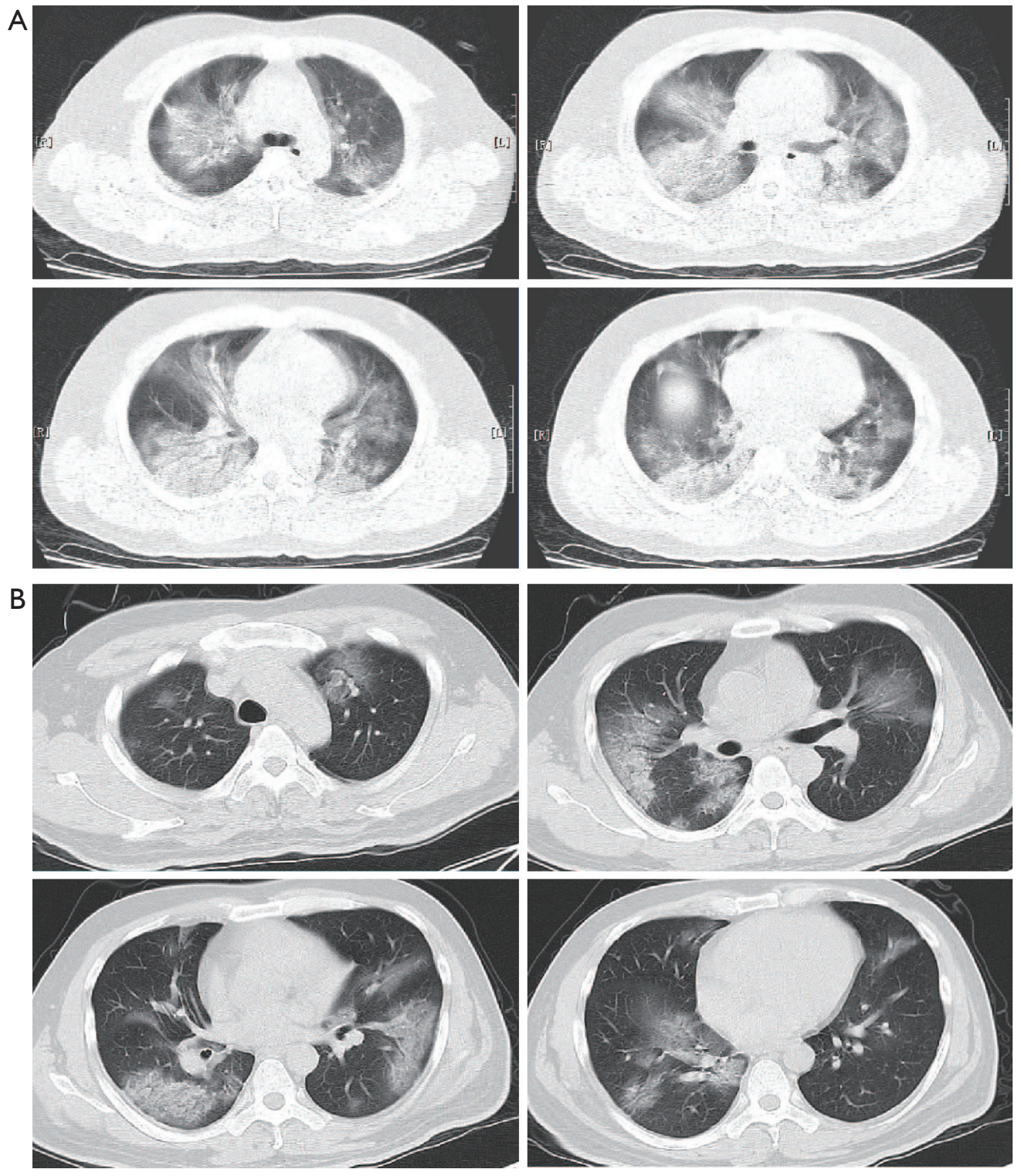

Figure 2 Chest computed tomography of critically ill COVID-19 patients. (A) In most critically severe cases, chest computed tomogram scans demonstrated bilateral diffuse ground-glass opacities, reticular pattern, and consolidation; (B) in most severe cases, the main patterns of abnormality on chest computed tomogram included localized ground-glass opacity of both lungs.

clinical characteristics, and treatment status of these patients, with the aim of providing a valuable reference for other colleagues.

Half of the critically ill patients in this study were $>60$ years old, which is similar to the findings from Zhejiang province and wuhan area $(4,7)$. Among the critically ill patients in Hebei, familial clusters accounted for almost half of cases, while none of these patients had a history of exposure to the Huanan seafood market. These results differ from findings in Wuhan but are similar to those in Zhejiang. The data in this study confirm significant human-to-human transmission of COVID-19 through close contact, which supports the restrictions introduced to minimize virus transmission by preventing people gathering in large groups. Similar to the findings of Huang et al., gender, age distribution, and exposure history were similar in the two groups, but patients with underlying diseases had a higher risk of developing critically severe disease (8). 
The rate of underlying diseases among critically ill patients, which is consistently predictive of a worse prognosis, was higher in Hebei than in Wuhan (5). Moreover, patients with critically severe COVID-19 had a longer duration from symptom onset to confirmation than those with severe disease.

Among the cardinal clinical symptoms like fever, cough, and expectoration was similar in both groups, while dyspnea was more common among patients with critically severe disease.

In terms of vital signs, the patients with critically severe COVID-19 had higher temperature, faster respiratory frequency, and more severe hypotension than those with severe disease, thus they required more respiratory and vasoactive support.

In patients with COVID-19, leucopenia and lymphopenia are common $(7,8)$. However, Huang et al. (8) found that ICU patients had higher WBC and inflammatory cytokine levels than non-ICU patients, indicating that the severity of COVID-19 is associated with cytokine storm. An autopsy study (9) reported that the counts of peripheral CD4+ and CD8+ cells were markedly decreased in critically ill patients with COVID-19. Our laboratory results showed similar results; critically severe patients had higher WBC, neutrophil percentage, and C-reactive protein and procalcitonin levels, and lower lymphocyte percentage, T cell subsets, and $\mathrm{PaO}_{2} / \mathrm{FiO}_{2}$, which indicated stronger inflammatory response and more severe lung injury. Severe immune dysfunction in patients with critically severe COVID-19 may result in them receiving more broadspectrum antibiotics. Patients in the critically severe group had mild coagulation disorders with slightly prolonged PT, and slightly elevated BUN and creatine kinase levels. Extrapulmonary complications, especially heart or kidney injury were common in the critically severe group, which was in line with the findings of previous studies $(7,8)$. Patients with critically severe disease also had higher APACHE II and SOFA scores $(7,8)$, and received more immunizing agents, albumin, and blood transfusions, as a result of the impact of the laboratory results.

Pan et al. (10) reported that only one or two lobes of the lung were damaged in early stages of COVID-19, with lung function remaining normal. As the disease progressed, the consolidations were gradually absorbed, resulting in ground-glass opacity or fiber-cord focus. In our study, chest CT imaging of patients with critically severe disease showed more ground-glass opacities, reticular pattern, and pulmonary edema, which is also similar with the findings of previous studies $(10,11)$. Pulmonary edema may result from a severe increase in capillary permeability.

All of the patients in our study received antiviral therapy. Lopinavir/ritonavir, interferon, and arbidol are recommended in Chinese Clinical Guidelines for COVID Pneumonia Diagnosis and Treatment (7th edition). Arbidol is also recommended as antiviral treatment for influenza infection in Russia and China (12). The effect of interferon has previously been evaluated in clinical studies on Middle East respiratory syndrome (MERS) and severe acute respiratory syndrome (SARS), but its benefits are still under discussion $(13,14)$. There are proven benefits of using traditional Chinese drugs to treat patients with mild COVID-19 (15), but their efficacy in the treatment of severe patients still needs to be studied.

Twenty (100\%) of the patients with critically severe disease and $26(83.9 \%)$ of the patients with severe disease received similar low doses of methylprednisolone, but the duration of treatment tended to be longer in the critically severe group. The use of corticosteroids for treating COVID-19 also presents a challenging problem. Because there is no clinical benefit in using corticosteroids to treat influenza, MERS, or SARS, the routine usage of corticosteroids for treating ARDS or shock associated with COVID-19 is not recommended by the World Health Organization (16). High-dose corticosteroids carry several potential risks, such as secondary infections, prolonged virus shedding, and long-term complications. However, the overwhelming inflammation in critically ill patients can lead their pneumonia to rapidly progress, and a physiologic or low dose of corticosteroids could alleviate the pulmonary fibrosis and prevent progressive pathological deterioration in patients with advanced ARDS, while also bringing earlier reversal of shock and a shorter length of ICU stay and mechanical ventilation (17-19). Moreover, a low-to-moderate dose of corticosteroids could reduce mortality in severe patients with influenza $\mathrm{A}\left(\mathrm{PaO}_{2} / \mathrm{FiO}_{2}\right.$ $<300 \mathrm{mmHg}$ ) (20). Thus, in China, low-dose and shortcourse methylprednisolone $(\leq 1-2 \mathrm{mg} / \mathrm{kg}$ per day, for 3-5 days) is recommended for patients with severe COVID-19. In addition, the use of corticosteroids should be cautiously applied in patients who have chronic hypoxemia or who receive regular corticosteroid therapy, due to underlying diseases (21).

Severe ARDS is the result of the pathophysiologic processes underlying severe COVID-19 infection, which is similar to SARS and MERS $(5,22-25)$. The majority of patients with severe COVID-19 presented with mild ARDS, 
and the patients with critically severe disease presented with more severe ARDS. Unfortunately, despite decades of research, the treatment of severe ARDS has remained focused on respiratory support, such as low tidal-volume mechanical ventilation, prone-position ventilation, and ECMO $(26,27)$. In our study, $70 \%$ of patients with critically severe COVID-19 received invasive mechanical ventilation, more than $50 \%$ received prone-position ventilation, and 2 received ECMO. Lower plasma $\mathrm{PCO}_{2}$ in patients with critically severe disease may indicate an unsatisfactory condition resulting from ventilator settings, sedation, or analgesia.

Fluid management is also important and challenging in ARDS. Compared with liberal fluid management, conservative fluid management may significantly decrease the severity of pulmonary edema, the duration of mechanical ventilation and ICU stay, and mortality in patients with ARDS $(28,29)$. Our results showed that patients with critically severe COVID-19 received more fluids and more diuretics than patients with severe disease, suggesting that it may be difficult to follow a conservative liquid management strategy because of the severity of the disease.

Clinical outcome was worse in patients with critically severe COVID-19; they had a mortality rate of $15 \%$, while no patients in the severe group died. Also, the length of hospital stay tended to be longer in the critically severe group. Further research is needed to explore the risk factors associated with worse outcome.

There are several limitations in the current study. First, the sample size of this study was small, which may only represent the characteristics of the patient population in Hebei, although it can also reflect some issues (e.g., characteristics of the patient group, treatment, etc.). Second, clinical data were limited in this retrospective study; thus, further evaluations in other regions need to be carried out.

\section{Conclusions}

Overall, patients with underlying diseases and longer confirmation times were more likely to develop to critically severe COVID-19. The patients in the critically severe group also had a higher risk of respiratory depression, circulatory collapse, extrapulmonary complications, and infection.

\section{Acknowledgments}

The authors wish to acknowledge all patients and their families involved in this study. We are grateful to the Expert Panel of the COVID-19 of Hebei Province and all health care workers around the world. We also thank Health Care Committee of Hebei Province for data support, and the Department of Science and Technology of Hebei Province for financial support.

Funding: This study was supported by the Department of Science and Technology of Hebei Province of China (20277707D).

\section{Footnote}

Reporting Checklist: The authors have completed the STROBE reporting checklist. Available at http://dx.doi. org/10.21037/apm-20-1273

Data Sharing Statement: Available at http://dx.doi. org/10.21037/apm-20-1273

Conflicts of Interest: All authors have completed the ICMJE uniform disclosure form (available at http://dx.doi. org/10.21037/apm-20-1273). The authors have no conflicts of interest to declare.

Ethical Statement: The authors are accountable for all aspects of the work in ensuring that questions related to the accuracy or integrity of any part of the work are appropriately investigated and resolved. All procedures performed in this study involving human participants were in accordance with the Declaration of Helsinki (as revised in 2013). Informed consent was taken from all the patients. This study was approved by the Ethics Commission of the Fourth Clinical Medical College of Hebei Medical University (2020KS002).

Open Access Statement: This is an Open Access article distributed in accordance with the Creative Commons Attribution-NonCommercial-NoDerivs 4.0 International License (CC BY-NC-ND 4.0), which permits the noncommercial replication and distribution of the article with the strict proviso that no changes or edits are made and the original work is properly cited (including links to both the formal publication through the relevant DOI and the license). See: https://creativecommons.org/licenses/by-nc-nd/4.0/.

\section{References}

1. Special Expert Group for Control of the Epidemic of 
Novel Coronavirus Pneumonia of the Chinese Preventive Medicine Association. An update on the epidemiological characteristics of novel coronavirus pneumonia COVID-19. Zhonghua Liu Xing Bing Xue Za Zhi 2020;41:139-44.

2. Wang D, Hu B, Hu C, et al. Clinical Characteristics of 138 Hospitalized Patients With 2019 Novel CoronavirusInfected Pneumonia in Wuhan, China. JAMA 2020;323:1061-9.

3. Diagnosis and Treatment of Pneumonia Caused by 2019$\mathrm{nCoV}$ (version 7), 2020 [cited $2020 \mathrm{Mar} 3$, in Chinese]. Available online: http://www.nhc.gov.cn/yzygj/s7653p/202 003/46c9294a7dfe4cef80dc7f5912eb1989.shtml

4. Xu XW, Wu XX, Jiang XG, et al. Clinical findings in a group of patients infected with the 2019 novel coronavirus (SARS-Cov-2) outside of Wuhan, China: retrospective case series. BMJ 2020;368:m606.

5. Yang X, Yu Y, Xu J, et al. Clinical course and outcomes of critically ill patients with SARS-CoV-2 pneumonia in Wuhan, China: a single-centered, retrospective, observational study. Lancet Respir Med 2020;8:475-81.

6. Zhang Z. Univariate description and bivariate statistical inference: the first step delving into data. Ann Transl Med 2016;4:91.

7. Zhang JJ, Dong X, Cao YY, et al. Clinical characteristics of 140 patients infected with SARS-CoV-2 in Wuhan, China. Allergy 2020;75:1730-41.

8. Huang C, Wang Y, Li X, et al. Clinical features of patients infected with 2019 novel coronavirus in Wuhan, China. Lancet 2020;395:497-506.

9. Xu Z, Shi L, Wang Y, et al. Pathological findings of COVID-19 associated with acute respiratory distress syndrome. Lancet Respir Med 2020;8:420-2.

10. Pan Y, Guan H. Imaging changes in patients with 2019nCov. Eur Radiol 2020;30:3612-3.

11. Pan Y, Guan H, Zhou S, et al. Initial CT findings and temporal changes in patients with the novel coronavirus pneumonia (2019-nCoV): a study of 63 patients in Wuhan, China. Eur Radiol 2020;30:3306-9.

12. Wang $Y$, Ding $Y$, Yang C, et al. Inhibition of the infectivity and inflammatory response of influenza virus by Arbidol hydrochloride in vitro and in vivo (mice and ferret). Biomed Pharmacother 2017;91:393-401.

13. Lau AC, Yam LY, So LK. Management of Critically Ill Patients with Severe Acute Respiratory Syndrome (SARS). Int J Med Sci 2004;1:1-10.

14. Arabi YM, Shalhoub S, Mandourah Y, et al. Ribavirin and Interferon Therapy for Critically Ill Patients With Middle
East Respiratory Syndrome: A Multicenter Observational Study. Clin Infect Dis 2020;70:1837-44.

15. Wang Z, Chen X, Lu Y, et al. Clinical characteristics and therapeutic procedure for four cases with 2019 novel coronavirus pneumonia receiving combined Chinese and Western medicine treatment. Biosci Trends 2020;14:64-8.

16. Russell CD, Millar JE, Baillie JK. Clinical evidence does not support corticosteroid treatment for 2019-nCoV lung injury. Lancet 2020;395:473-5.

17. Marik PE. Steroids for sepsis: yes, no or maybe. J Thorac Dis 2018;10:S1070-S1073.

18. Zhou W, Liu Y, Tian D, et al. Potential benefits of precise corticosteroids therapy for severe 2019-nCoV pneumonia. Signal Transduct Target Ther 2020;5:18.

19. Biffl WL, Moore FA, Moore EE, et al. Are corticosteroids salvage therapy for refractory acute respiratory distress syndrome? Am J Surg 1995;170:5915; discussion 595-6.

20. Li H, Yang SG, Gu L, et al. Effect of low-to-moderatedose corticosteroids on mortality of hospitalized adolescents and adults with influenza A(H1N1)pdm09 viral pneumonia. Influenza Other Respir Viruses 2017;11:345-54.

21. Shang L, Zhao J, Hu Y, et al. On the use of corticosteroids for 2019-nCoV pneumonia. Lancet 2020;395:683-4.

22. Arabi YM, Al-Omari A, Mandourah Y, et al. Critically Ill Patients With the Middle East Respiratory Syndrome: A Multicenter Retrospective Cohort Study. Crit Care Med 2017;45:1683-95.

23. Arabi YM, Arifi AA, Balkhy HH, et al. Clinical course and outcomes of critically ill patients with Middle East respiratory syndrome coronavirus infection. Ann Intern Med 2014;160:389-97.

24. Kalil AC, Thomas PG. Influenza virus-related critical illness: pathophysiology and epidemiology. Crit Care 2019;23:258.

25. Lew TW, Kwek TK, Tai D, et al. Acute respiratory distress syndrome in critically ill patients with severe acute respiratory syndrome. JAMA 2003;290:374-80.

26. Fan E, Brodie D, Slutsky AS. Acute Respiratory Distress Syndrome: Advances in Diagnosis and Treatment. JAMA 2018;319:698-710.

27. Qiu HB, Li XY, Du B, et al. The keypoints in treatment of the critical novel coronavirus pneumonia patient. Zhonghua Jie He He Hu Xi Za Zhi 2020;43:273-7.

28. Warren MA, Zhao Z, Koyama T, et al. Severity scoring of lung oedema on the chest radiograph is associated with 
clinical outcomes in ARDS. Thorax 2018;73:840-6.

29. Silversides JA, Major E, Ferguson AJ, et al. Conservative fluid management or deresuscitation for patients with

sepsis or acute respiratory distress syndrome following the resuscitation phase of critical illness: a systematic review and meta-analysis. Intensive Care Med 2017;43:155-70.

(English Language Editor: J. Reynolds)

Cite this article as: Chen $\mathrm{Y}$, Zhang K, Zhu G, Liu L, Yan X, Cai Z, Zhang Z, Zhi H, Hu Z. Clinical characteristics and treatment of critically ill patients with COVID-19 in Hebei. Ann Palliat Med 2020;9(4):2118-2130. doi: 10.21037/ apm-20-1273 